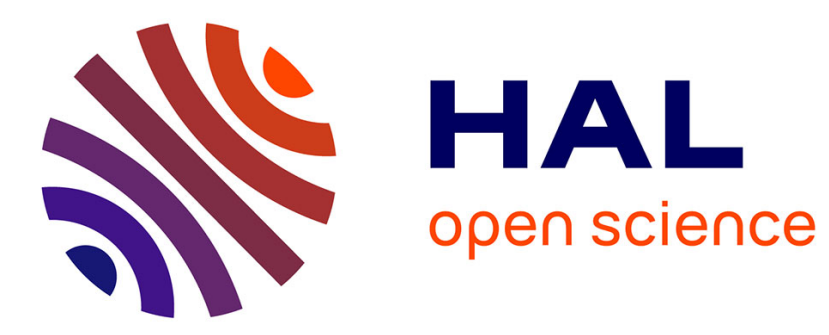

\title{
Brillouin light scattering study of glassy sorbitol
}

\author{
Beatrice Ruta, Giulio Monaco, Daniele Fioretto, Filippo Scarponi
}

\section{To cite this version:}

Beatrice Ruta, Giulio Monaco, Daniele Fioretto, Filippo Scarponi. Brillouin light scattering study of glassy sorbitol. Philosophical Magazine, 2008, 88 (33-35), pp.3939-3946. 10.1080/14786430802317586 . hal-00513932

\section{HAL Id: hal-00513932 \\ https://hal.science/hal-00513932}

Submitted on 1 Sep 2010

HAL is a multi-disciplinary open access archive for the deposit and dissemination of scientific research documents, whether they are published or not. The documents may come from teaching and research institutions in France or abroad, or from public or private research centers.
L'archive ouverte pluridisciplinaire HAL, est destinée au dépôt et à la diffusion de documents scientifiques de niveau recherche, publiés ou non, émanant des établissements d'enseignement et de recherche français ou étrangers, des laboratoires publics ou privés. 


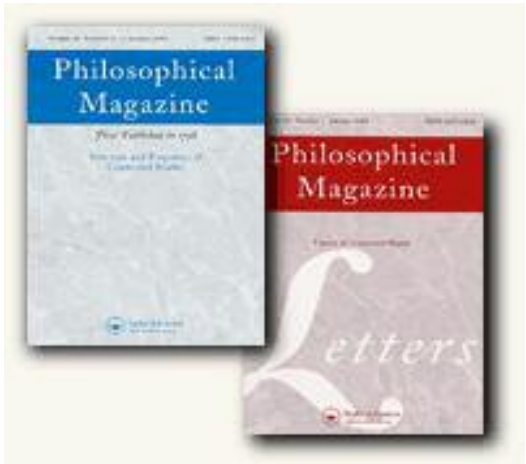

Brillouin light scattering study of glassy sorbitol

\begin{tabular}{|r|l|}
\hline Journal: & Philosophical Magazine \& Philosophical Magazine Letters \\
\hline Manuscript ID: & TPHM-08-May-0189 \\
\hline Journal Selection: & Philosophical Magazine \\
\hline Date Submitted by the & 23-May-2008 \\
\hline Complete List of Authors: & $\begin{array}{l}\text { Ruta, Beatrice; European Synchrotron Radiation Facility } \\
\text { Monaco, Giulio; European Synchrotron Radiation Facility } \\
\text { Kioretto, Daniele; Università di Perugia, Fisica; CNR-INFM CRS Soft } \\
\text { Scarponi, Filippo; Università di Perugia, Fisica; CNR-INFM CRS Soft }\end{array}$ \\
\hline Keywords: & glass, vibrational properties, vibrational spectroscopy \\
\hline Keyser supplied): & glass, vibrational properties, vibrational spectroscopy \\
\hline Note: The following files were submitted by the author for peer review, but cannot be converted \\
to PDF. You must view these files (e.g. movies) online. \\
\hline articolo_last.tex
\end{tabular}

\section{(5) ScholaroNE \\ Manuscript Central}




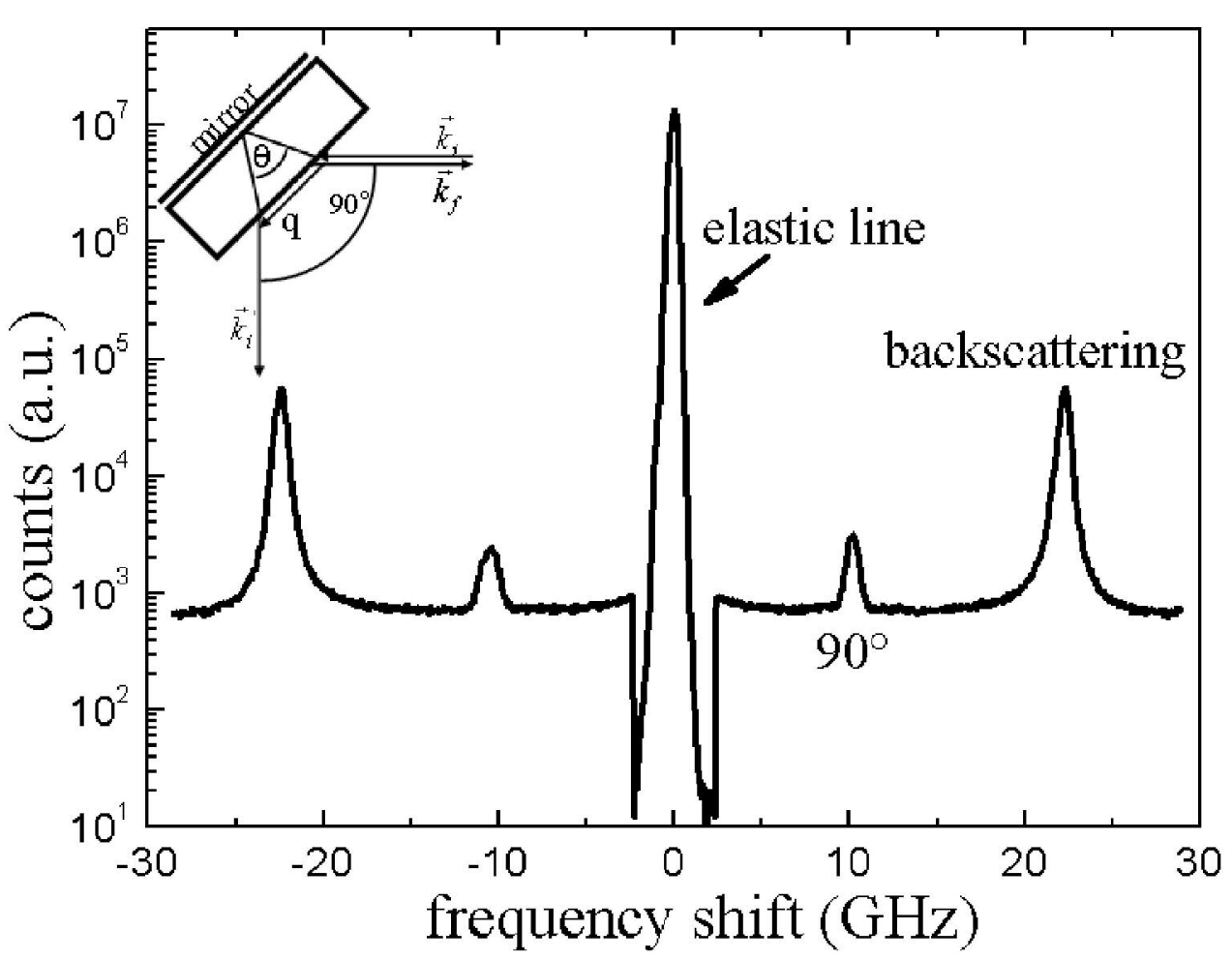

Brillouin light scattering spectrum of glassy sorbitol at $T=281 \mathrm{~K}$. The elastic line, that can be considered to a good approximation as the instrumental function, is here presented on a y-scale different from that of the inelastic signal. Two Brillouin doublets are present, corresponding to the two different scattering angles $\theta_{\text {probed at the same time as }}$ schematically shown in the inset. The incident and scattered beams are represented by the arrows $k_{i}$ and $k_{f}$ for, and by $k_{i}$ and $k_{f}$ for $122 \times 95 \mathrm{~mm}(600 \times 600 \mathrm{DPI})$ 


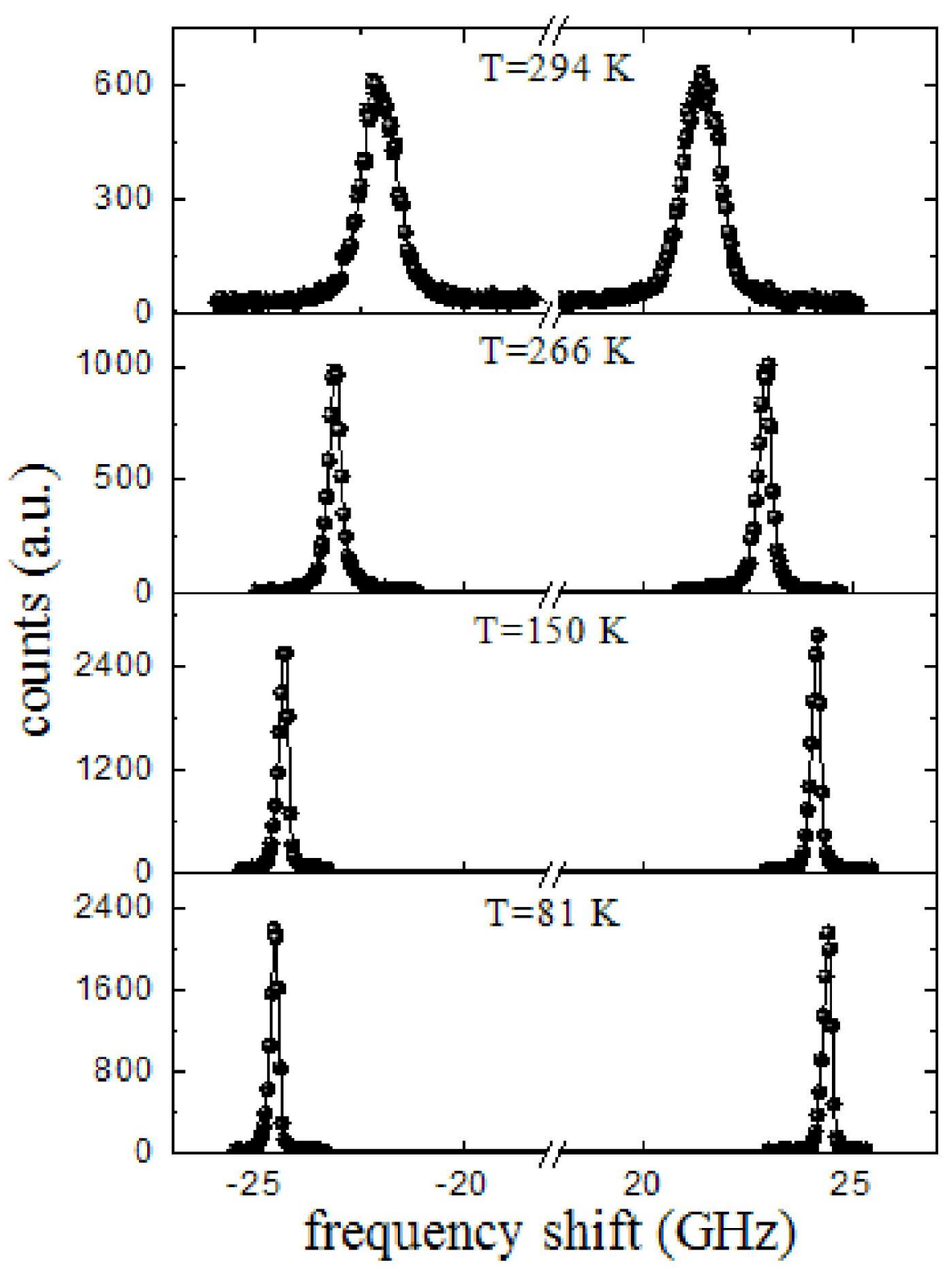

Brillouin light scattering spectra of sorbitol at the indicated temperatures collected in the backscattering configuration.

$100 \times 132 \mathrm{~mm}(600 \times 600 \mathrm{DPI})$ 


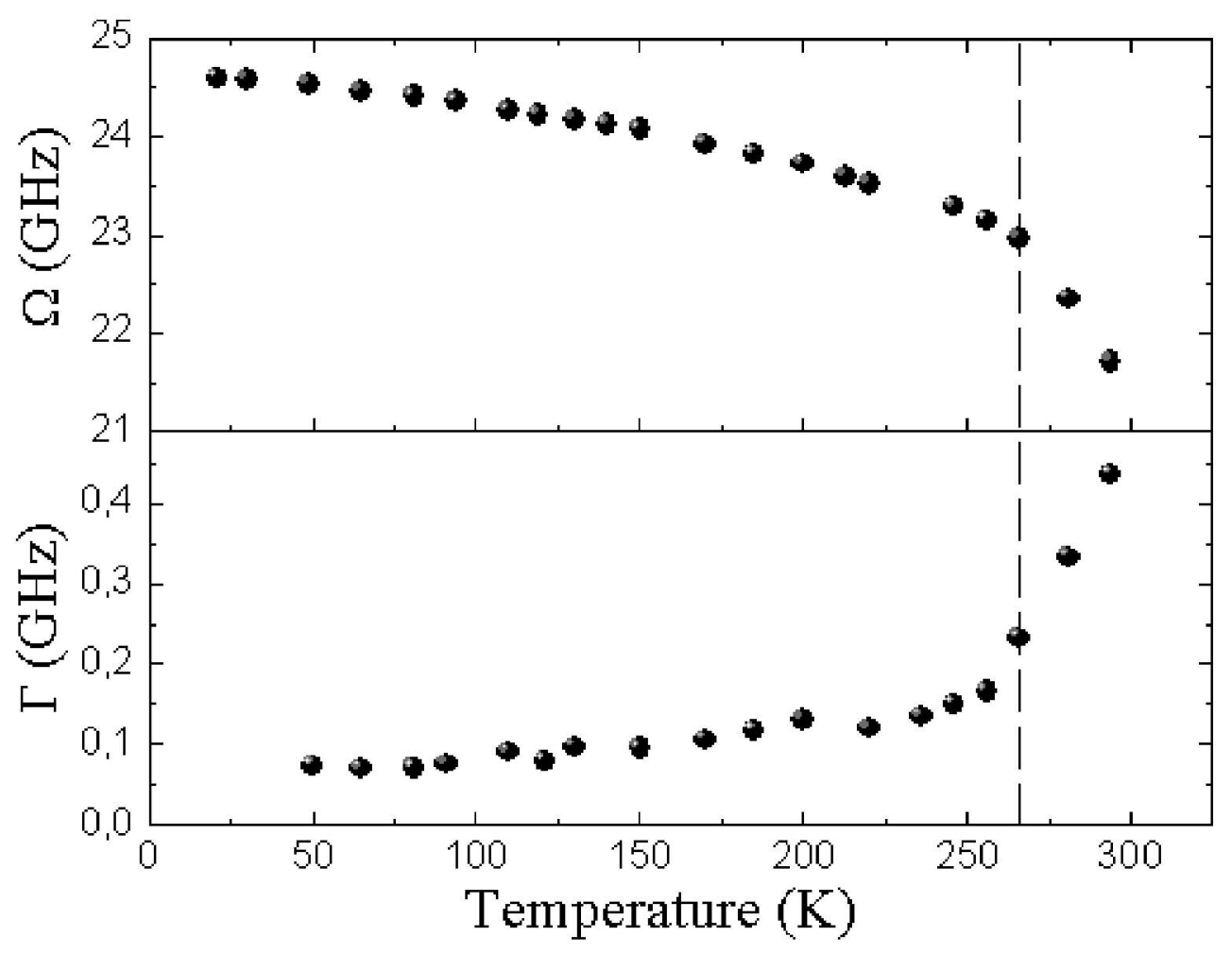

Temperature dependence of the frequency shift $\Omega$ (top panel) and of the linewidth $\Gamma$ (bottom panel) of the longitudinal acoustic modes. The dashed line indicates $T_{g}=266 \mathrm{~K}$. $106 \times 82 \mathrm{~mm}(600 \times 600 \mathrm{DPI})$ 


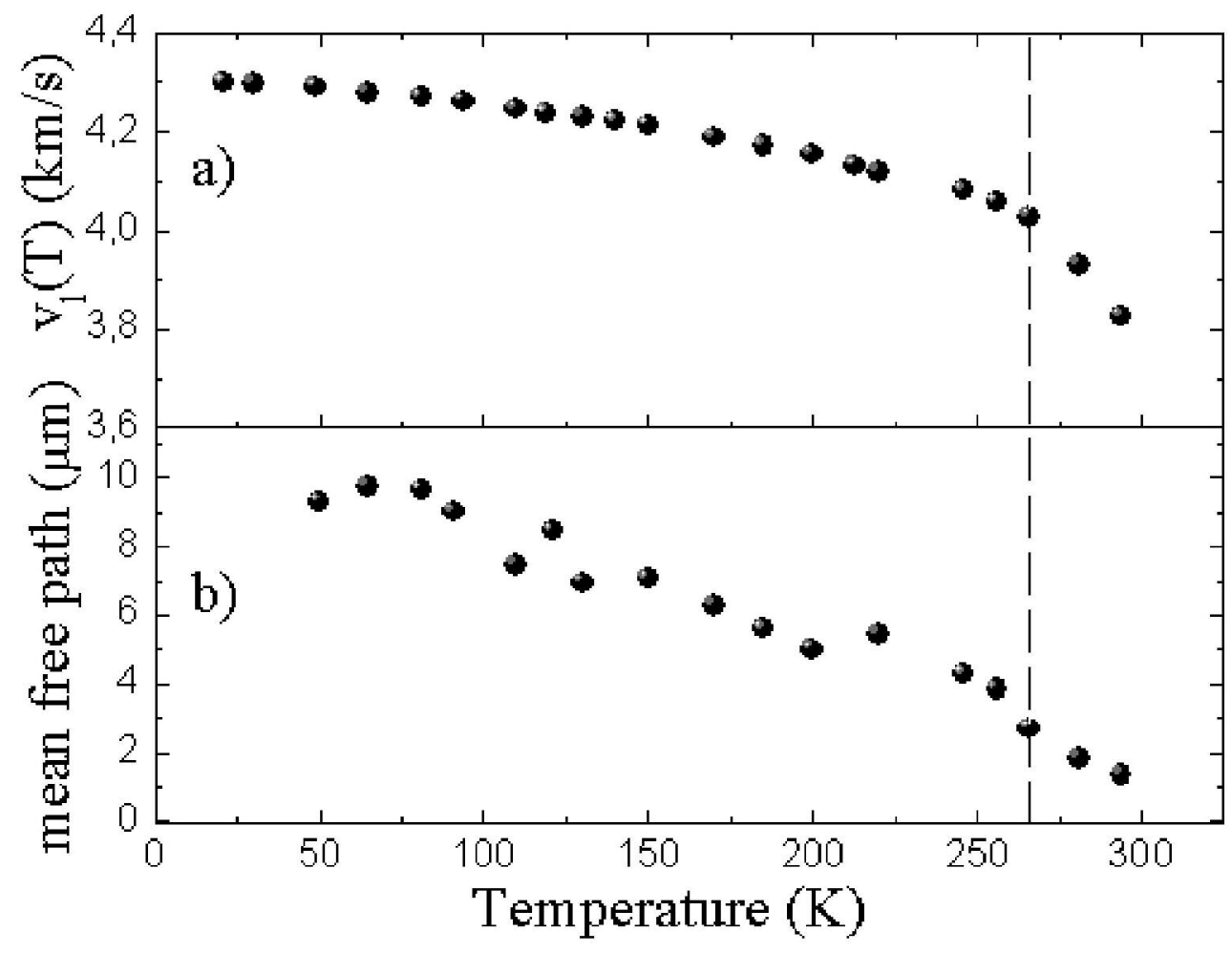

Temperature dependence of the sound velocity $v_{I}(a)$ and of the mean free path $I(b)$ of the longitudinal acoustic modes. The dashed line indicates $T_{g}=266 \mathrm{~K}$ $105 \times 80 \mathrm{~mm}(600 \times 600$ DPI $)$ 


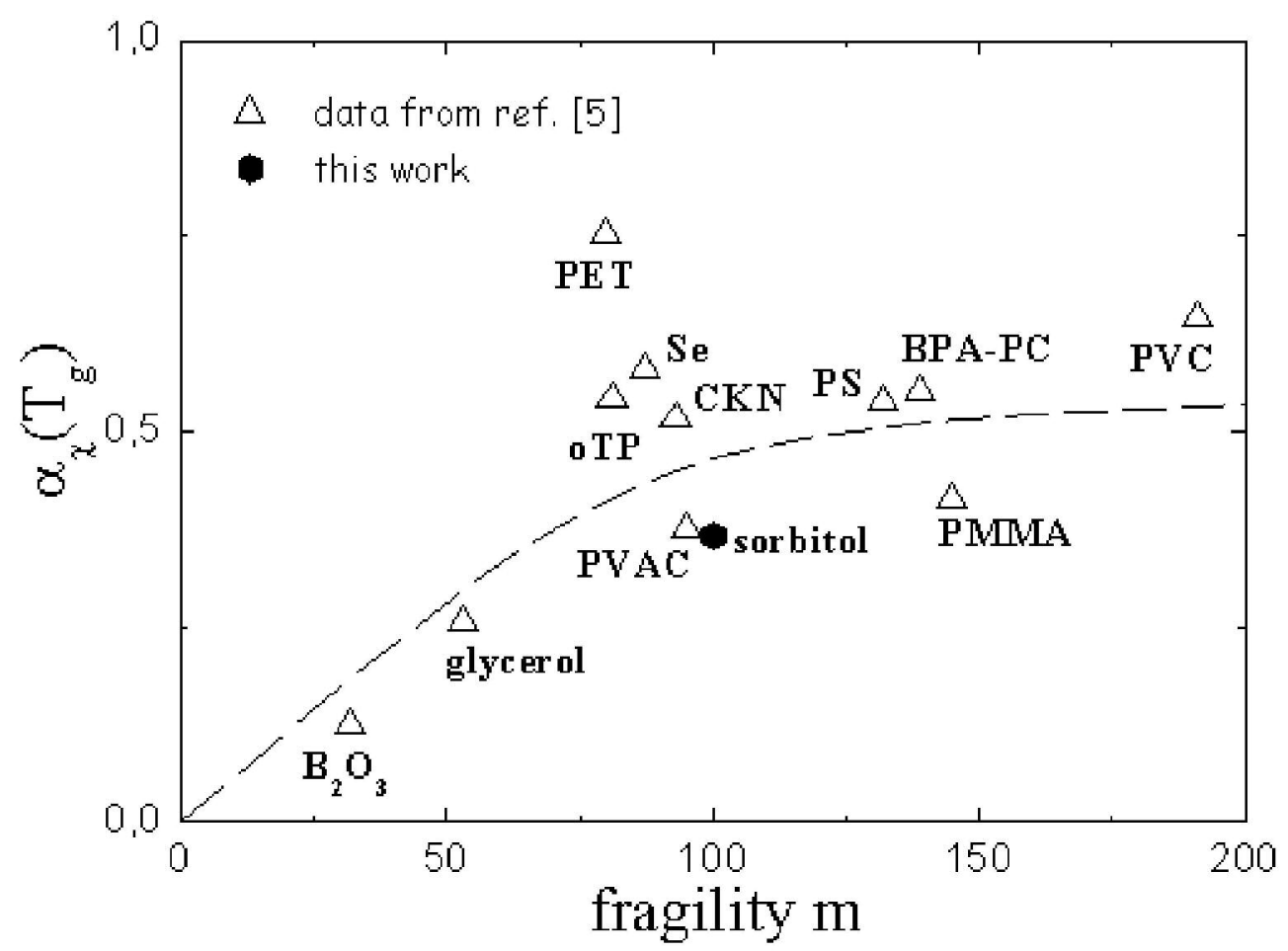

Correlation between the ratio $\alpha \chi$ of vibrational to relaxational compressibility at $\mathbf{T}_{\mathrm{g}}$ and the fragility $\mathrm{m}$. The empty symbols are taken from [5], while the full circle corresponds to sorbitol. The dashed line is a guide for the eye. $105 \times 77 \mathrm{~mm}(600 \times 600 \mathrm{DPI})$ 


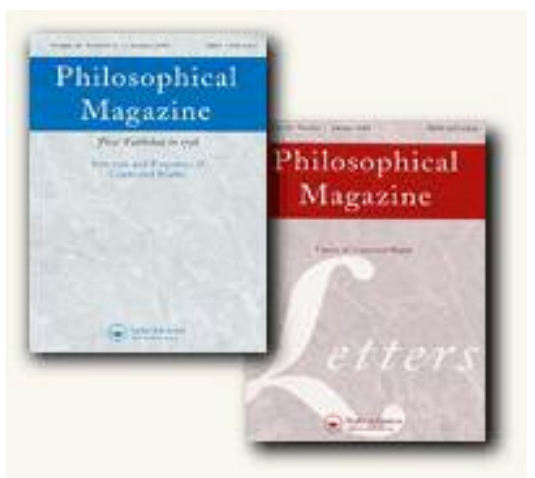

Brillouin light scattering study of glassy sorbitol

\begin{tabular}{|r|l|}
\hline Journal: & Philosophical Magazine \& Philosophical Magazine Letters \\
\hline Manuscript ID: & TPHM-08-May-0189 \\
\hline Journal Selection: & Philosophical Magazine \\
\hline Aute Submitted by the & 23-May-2008 \\
\hline Complete List of Authors: & $\begin{array}{l}\text { Ruta, Beatrice; European Synchrotron Radiation Facility } \\
\text { Monaco, Giulio; European Synchrotron Radiation Facility } \\
\text { Fioretto, Daniele; Università di Perugia, Fisica; CNR-INFM CRS Soft } \\
\text { Scarponi, Filippo; Università di Perugia, Fisica; CNR-INFM CRS Soft }\end{array}$ \\
\hline Keywords: & glass, vibrational properties, vibrational spectroscopy \\
\hline Keywords (user supplied): & glass, vibrational properties, vibrational spectroscopy \\
\hline & \multicolumn{2}{|l}{} \\
\hline
\end{tabular}

\section{5cholarone \\ Manuscript Central}


Philosophical Magazine

Vol. 00, No. 00, 15 May 2008, 1-8

\title{
Brillouin light scattering study of glassy sorbitol
}

\author{
B. Ruta $\dagger^{*}$, G. Monaco†, F. Scarponił $₫$, D. Fioretto $₫$ \\ †European Synchrotron Radiation Facility, BP220, F-38043 Grenoble, France \\ $\ddagger$ Dipartimento di Fisica, Univerisità di Perugia, Via A. Pascoli, I-06100 Perugia, Italy \\ ๑CNR-INFM CRS Soft, Univerisità di Roma "La Sapienza", P. A. Moro 2, I-00185 \\ Roma, Italy
}

\begin{abstract}
Brillouin light scattering has been used to investigate the density fluctuations of the fragile glass-former sorbitol in the $\mathrm{GHz}$ frequency range and at temperatures between 20 and 294 K. Longitudinal acoustic sound velocity and attenuation data are here reported and discussed. These data are used to test the existence of a correlation between elastic properties of glasses and viscous properties of the corresponding deeply undercooled melts above the glass transition temperature, $T_{g}$. In particular, and as for other fragile systems, the ratio of the vibrational to the relaxational compressibility at $T_{g}$ is found to be basically independent of fragility.
\end{abstract}

Keywords: Glasses; acoustic properties; light scattering.

\section{Introduction}

An interesting open question in the physics of disordered systems is whether there exists a connection between the high-frequency elastic properties of glasses and the viscous properties of the corresponding deeply undercooled melts above the glass transition temperature, $T_{g}$. The latter ones are commonly considered to be efficiently grasped by the fragility, which in its kinetic definition is a number that quantifies how fast the structural relaxation time, or the shear viscosity, increases with decreasing temperature on approaching $T_{g}[1]$.

For example, some years ago Scopigno et al. proposed a correlation between the kinetic fragility, $m$, and a parameter $\alpha$ that describes the temperature dependence of the long-wavelength limit of the non-ergodicity factor in the glassy phase [2]. Within the harmonic approximation, $\alpha$ only depends on the vibrational properties of the system in its inherent structure. Specifically, $\alpha$ was suggested to be directly proportional to the kinetic fragility, thus establishing a direct link between the vibrational properties of glasses and the fragility of the corresponding melts. After this work, a few glasses that do not follow the proposed linear relation have been identified [3]; however, the basic idea of a correlation between elastic properties and fragility remains at least qualitatively valid [3-5], even though none of the proposed correlations seems to hold for all glasses at a quantitative level.

This article reports Brillouin light scattering (BLS) measurements on sorbitol. This system is a very fragile glass-former that is however characterized by the

*Corresponding author. Email: ruta@esrf.fr 
presence of hydrogen bonds. Its high fragility ( $\mathrm{m} \sim 100[6])$, typically only found in polymeric systems, makes sorbitol an excellent molecular candidate to test the validity of the proposed correlations between the fragility and the elastic behavior of glasses.

\section{Experimental details}

Sorbitol $\left(\mathrm{C}_{6} \mathrm{H}_{14} \mathrm{O}_{6}, T_{g}=266 \mathrm{~K}\right.$, melting temperature $\left.T_{m}=368 \mathrm{~K}[7]\right)$ of $\geq 99 \%$ purity was purchased from Sigma-Aldrich Chemicals and kept for two days under vacuum at about $383 \mathrm{~K}$ to obtain an homogeneous and well transparent liquid, free from any gas content. Two samples were prepared. The first one was obtained from a liquid sorbitol film of about $80 \mu \mathrm{m}$ placed into a container with an optically polished silicon plate on the bottom and closed with a quartz window on the top. The sample glass was prepared by quenching the liquid film in mixed ice and salt $(\mathrm{T} \sim 255 \mathrm{~K})$. The whole sample preparation was performed in a nitrogen atmosphere to avoid contamination with water. The second glass sample was prepared as the first one but using this time as holder a glass tube with an inner diameter of $2 \mathrm{~mm}$ and an outer diameter of $6 \mathrm{~mm}$.

The experiment was performed using the beam of a diode pumped solid state laser operating on a single mode of the $532 \mathrm{~nm}$ line and vertically polarized. The light scattered from the sample in the horizontal plane was analysed by means of a Sandercock type $(3+3)$-pass tandem Fabry-Perot interferometer, with a typical contrast higher than $10^{9}$ and a finesse of $\sim 100$. The width of the instrumental function and the free spectral range can be modified by changing the distance between the mirrors in the interferometer. Brillouin spectra were collected in the temperature range 20-294 K, and a Cryomech ST405 cryostat was used to regulate the temperature. The measurements were taken both on cooling and on heating the sample, without any polarization analyser (VU configuration).

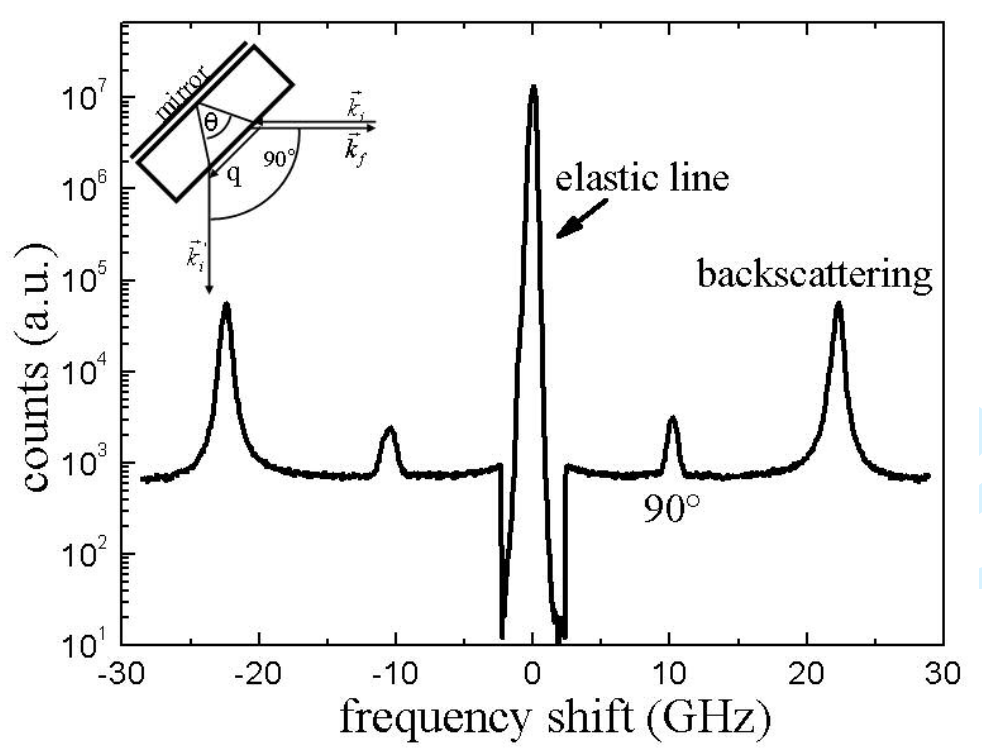

Figure 1. Brillouin light scattering spectrum of glassy sorbitol at $\mathrm{T}=281 \mathrm{~K}$. The elastic line, that can be considered to a good approximation as the instrumental function, is here presented on a y-scale different from that of the inelastic signal. Two Brillouin doublets are present, corresponding to the two different scattering angles $\theta$ probed at the same time as schematically shown in the inset. The incident and scattered beams are represented by the arrows $\overrightarrow{k_{i}}$ and $\overrightarrow{k_{f}}$ for $\theta=180^{\circ}$, and by $\overrightarrow{k_{i}^{\prime}}$ and $\overrightarrow{k_{f}}$ for $\theta=90^{\circ}$. 
A first set of measurements was performed using the sorbitol film as sample and using a distance of $d=5 \mathrm{~mm}$ between the mirrors in the interferometer in order to obtain a good luminosity while keeping the instrumental resolution to a still reasonably good level $(\approx 380 \mathrm{MHz}$ FWHM). The choice of the experimental setup was determined by the interest to measure the momentum transferred in the scattering process. In fact the exchanged momentum $q$ is given by $q=(4 \pi n / \lambda) \sin (\theta / 2)$, where $\theta$ is the scattering angle, $\lambda$ the incident wavelength of the laser source and $n$ the refractive index of the sample. However, to the best of our knowledge no published data of the refractive index of glassy sorbitol are available. We then chose a configuration that allowed us to measure $q$ as well. In fact, as shown in the inset of Figure 1, putting the sample at $45^{\circ}$ with respect to the incident beam and measuring the backscattered radiation it is possible to simultaneously probe two scattering geometries [8]: (i) the backscattering geometry itself, with transferred momentum $q_{b a c k}=\left|\overrightarrow{k_{f}}-\overrightarrow{k_{i}}\right|=4 \pi n / \lambda$, and (ii) the $90^{\circ}$ scattering angle geometry, allowed by the fact that the beam reflected by the mirror behind the sample acts as a second incident beam with wavenumber $\overrightarrow{k_{i}^{\prime}}$, and in this case the exchanged momentum is $q_{90^{\circ}}=\left|\overrightarrow{k_{f}}-\overrightarrow{k_{i}^{\prime}}\right|=4 \pi /(\lambda \sqrt{2})$.

An example of a spectrum obtained with this configuration is shown in Figure 1. If we assume that no dispersion in the sound velocity takes place between the two probed q values, the ratio of the Brillouin frequency positions $\nu$ of the two Stokes or anti-Stokes peaks directly gives the refractive index:

$$
n=\frac{\nu_{b a c k}}{\nu_{90^{\circ}}} \frac{\sqrt{2}}{2}
$$

This scattering configuration was used in the temperature range 150-294 $\mathrm{K}$ to obtain the refractive index of glassy sorbitol. At temperatures lower than about $150 \mathrm{~K}$, the sample cracked, thus leading to a large increase in the stray light that made the measurements of the refractive index impossible. A second set of measurements was then performed in the 20-294 K temperature range using only the standard backscattering configuration on both the prepared samples, in order to measure accurately the position and the width of the Brillouin peaks. In this case a distance of $d=17 \mathrm{~mm}$ between the mirrors in the interferometer was employed to obtain an instrumental resolution of $\approx 140 \mathrm{MHz}$. For each temperature various measurements were taken on both heating and cooling the sample to check the reproducibility of the data. No differences were found neither among the different sets of data nor between the two samples.

\section{Results and discussion}

From the first set of measurements we obtained for the refractive index $n$ the value $1.52 \pm 0.01$, basically constant in the $150-294 \mathrm{~K}$ temperature range. In order to evaluate the temperature dependence of $n$ down to the lowest temperatures investigated in the second set of measurements, we estimated $n$ using the ClausiusMossotti relation together with density data estrapolated from those reported in Ref. [9].

Figure 2 shows some examples of BLS spectra of sorbitol collected in the backscattering geometry (second set of measurements) for different temperatures above and below the glass transition temperature. For all spectra the Brillouin doublet corresponding to the longitudinal acoustic modes is shown. Two features 
are immediately evident: the rapid decrease of the width of the Brillouin peaks and the clear shift of their position toward higher frequencies on decreasing the temperature.

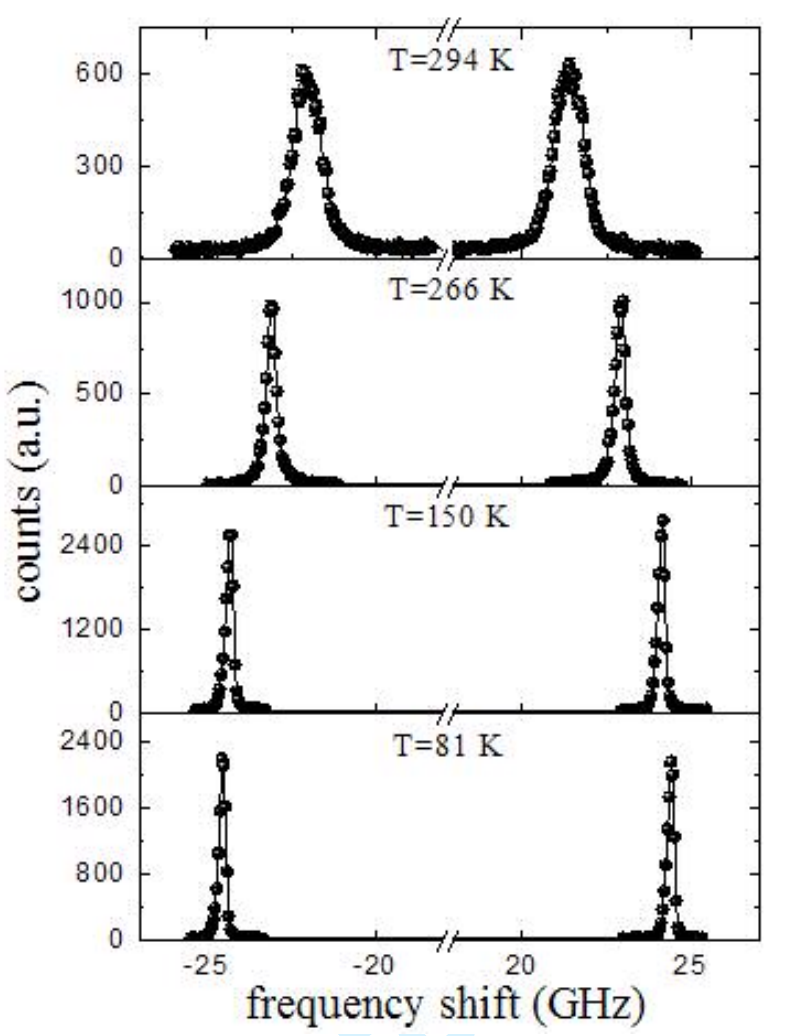

Figure 2. Brillouin light scattering spectra of sorbitol at the indicated temperatures collected in the backscattering configuration.

To determine the frequency position $\Omega$ and the full width at half maximum $\Gamma$ of the longitudinal acoustic modes, the spectra were fitted in a narrow frequency range around the Brillouin peak using a damped harmonic oscillator model function

$$
I(Q, \omega)=I_{0} \frac{\Gamma \Omega^{2}}{\left(\omega^{2}-\Omega^{2}\right)^{2}+(\Gamma \omega)^{2}}
$$

convoluted to the instrumental resolution. The values of $\Omega$ and $\Gamma$ obtained by this fitting procedure are reported in Figure 3. There is a clear change of slope in the temperature dependence of the Brillouin peak position that comes together with a rapid increase of the linewidth when crossing $T_{g}$. Such features are typically found in glass-formers; in particular, the second one is due to the structural relaxation entering, above $T_{g}$, the frequency window explored by BLS.

From the linear behavior of $\Omega$ close to $T_{g}$ (both in the glass and in the undercooled liquid) we estimated the Grüneisen parameter $\gamma_{G}$ through the relation [10]: $\gamma=$ $-(\partial \ln (\Omega) / \partial \ln (V))=-\left.\left(1 / \alpha_{P}\right)(\partial \ln (\Omega) / \partial T)\right|_{V}$, where $V$ is the volume and $\alpha_{P}$ the isobaric thermal expansion coefficient that we derived from the available PVT data [9]. This Grüneisen parameter reflects the role of anharmonicity on the acoustic excitations, and comes out to be equal to $3.6 \pm 1.1$ in the glass and to $4.7 \pm 0.3$ in the liquid phase. It is interesting to observe that these two values are basically compatible within errorbars, thus indicating that in good approximation the change of slope observed in the temperature dependence of the Brillouin frequency in 
Fig. 3 directly reflects the corresponding change in the slope of the temperature dependence of the density.

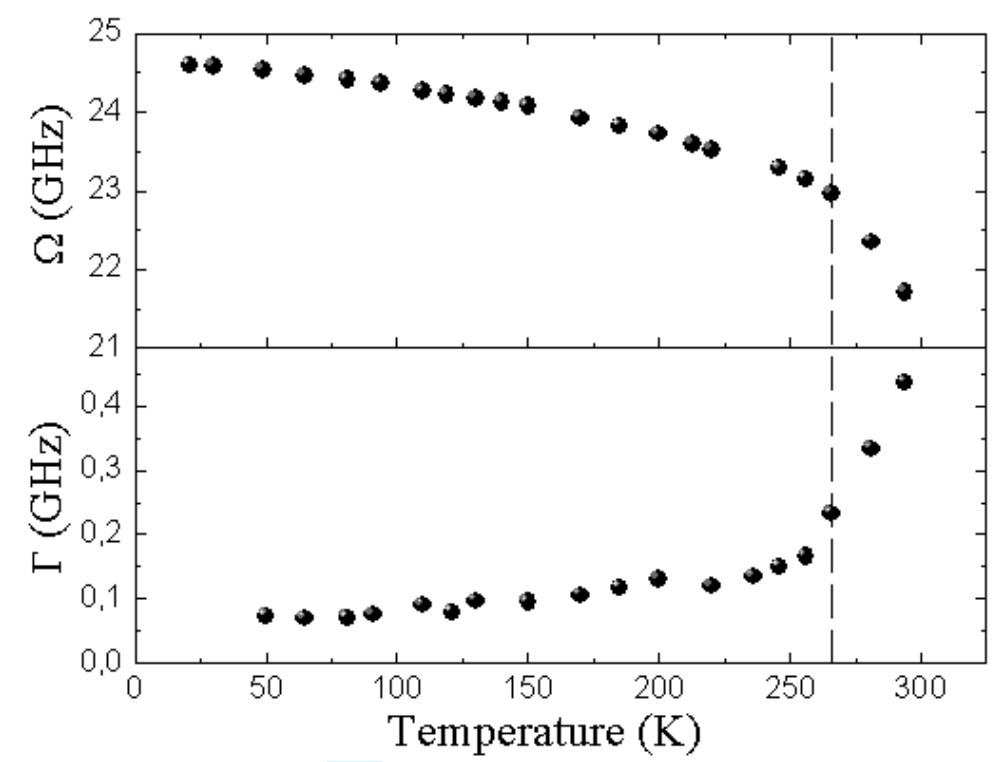

Figure 3. Temperature dependence of the frequency shift $\Omega$ (top panel) and of the linewidth $\Gamma$ (bottom panel) of the longitudinal acoustic modes. The dashed line indicates $T_{g}=266 \mathrm{~K}$.

In the simple Akhiezer model of anharmonicity the Grüneisen parameter enters in the description of the linewidth of the longitudinal acoustic waves through the relation:

$$
\Gamma=\gamma_{G}^{2} \frac{c_{V} T v}{2 v_{D}^{3}} \Omega^{2} \tau_{t h}
$$

where $c_{V}$ is the constant volume specific heat, $v$ the longitudinal sound velocity, $v_{D}$ the Debye velocity and $\tau_{t h}$ an average relaxation time [11, 12]. The thermodynamic quantities present in this equation have been taken from Refs. [9, 13], while the Debye sound velocity has been calculated by estimating the transverse sound velocity as explained later in the text. Eq. 3 can be used to calculate the relaxation time $\tau_{t h}$ which turns out to be almost temperature independent in the glassy phase with an average value of $\tau_{t h}=0.09 \mathrm{ps}$. This value is similar to that estimated in other systems such as OTP and $\mathrm{SiO}_{2}[11,14]$.

The frequency position and the linewidth of the Brillouin peaks can be used to obtain the real part $\left(M^{\prime}\right)$ and imaginary part $\left(M^{\prime \prime}\right)$ of the longitudinal elastic modulus $M$, through the relations $M^{\prime}(\Omega)=\Omega^{2} \rho / q^{2}$ and $M^{\prime \prime}(\Omega)=\Gamma \Omega \rho / q^{2}$, where $\rho$ is the density. Alternatively, the same information can be reported in terms of the sound velocity $v_{l}=\sqrt{M^{\prime} / \rho}=\Omega / q$ and of the mean free path $l=v_{l} / \Gamma$ of the longitudinal acoustic excitations, as shown in figure 4 (a) and (b), respectively. In the glassy phase the longitudinal sound velocity reaches values higher than $\sim 4000 \mathrm{~m} / \mathrm{s}$ that are quite exceptional for a fragile system such as sorbitol. We can estimate as well the transverse sound velocity $v_{t}=\sqrt{G^{\prime} / \rho}$, where $G^{\prime}$ is the shear modulus. In general in a glass $G^{\prime}$ is given by $G^{\prime}=\frac{3}{4}\left[M^{\prime}-B^{\prime}\right]$ where $B^{\prime}$ is the real part of the bulk modulus. The bulk modulus, in turn, can be calculated from the available PVT and calorimetric literature data $[9,13]$, being $B^{\prime}=\gamma / \chi_{T \infty}$, where $\chi_{T \infty}$ is the high-frequency isothermal compressibility and $\gamma=c_{P} / c_{V}$ is the 


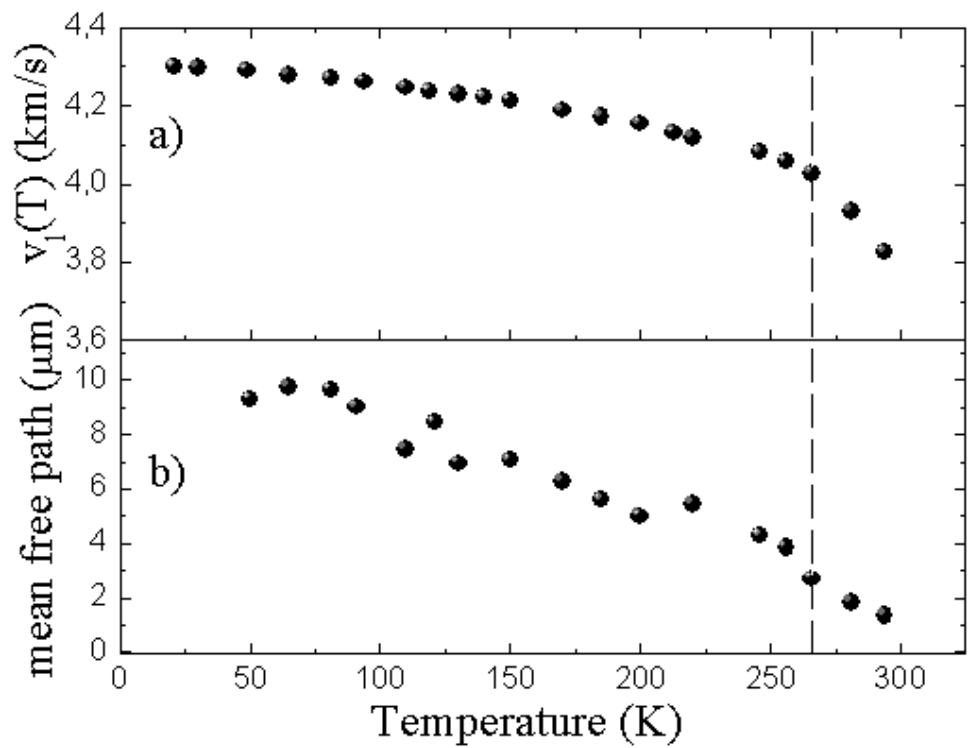

Figure 4. Temperature dependence of the sound velocity $v_{l}$ (a) and of the mean free path $l$ (b) of the longitudinal acoustic modes. The dashed line indicates $T_{g}=266 \mathrm{~K}$

constant pressure to constant volume specific heat ratio. From these relations we calculate a transverse sound velocity of $2300 \mathrm{~m} / \mathrm{s}$ at $T_{g}$. We have thus available the information on the two independent elastic moduli of the sorbitol glass.

The knowledge of the elastic moduli of glassy sorbitol can be used to test some of the proposed correlations between elastic properties of glasses and fragility. For example, Novikov and coworkers found that the weaker a glass resists to shear stress in comparison to the bulk one (higher $B^{\prime} / G^{\prime}$ ratio), the more fragile is the behavior of the corresponding melt $[3,4]$. This correlation is quantified by the relation $m=29\left(B^{\prime} / G^{\prime}-0.41\right)[3,4]$. In the case of glassy sorbitol $B^{\prime} / G^{\prime}=1.73$, which would lead to $m=38$ according to the previous relation. This value is in clear disagreement with the one obtained using dielectric measurements in the supercooled liquid $(m \sim 100)$ [6]. The existence of systems showing strong deviations from the previously recalled simple linear relation was underlined already in Refs. [3, 4], such for instance polystyrene. Novikov and coworkers suggested that the systems showing such deviations might be those who show differences between the kinetic and the thermodynamic fragility [15] . In fact, in the case of polystyrene the correlation between the elastic moduli and fragility seems to be recovered once the thermodynamic fragility is considered [3, 4]. However, this explanation does not seem to hold for the case of glassy sorbitol where the two fragilities were found to be very similar [6]. In other words, sorbitol really seems to be a peculiar system: from the elasticity point of view it shows a behavior similar to that of strong glasses, while it presents at the same time features of fragile systems, i.e. the strong departure of the structural relaxation time from the Arrhenius behavior above $T_{g}$.

Following Buchenau and Wischnewski [5] we can introduce a vibrational compressibility $\chi_{v}=1 / M^{\prime}$ and we can estimate the ratio $\alpha_{\chi}$ between the vibrational and relaxational compressibility:

$$
\alpha_{\chi}\left(T_{g}\right)=\frac{\chi_{v}\left(T_{g}\right)}{\chi_{T}\left(T_{g}\right)-\chi_{v}\left(T_{g}\right)}
$$

where $\chi_{T}$ is the isothermal compressibility measured in the liquid phase close to $T_{g}$. As far as the harmonic approximation holds to calculate the non-ergodicity 
factor up to $T_{g}$, this ratio coincides with the $\alpha$ parameter introduced by Scopigno et al. [2] and which was proposed to be simply proportional to the kinetic fragility through the relation $m=135 \alpha$. In the present case, we find $\alpha_{\chi}=0.367$, which given the known fragility of sorbitol - is a value too low to fulfill the proposed linear relation. However, as previously mentioned, the reason of the discrepancy might well lie in the fact that the harmonic approximation does not hold to describe the temperature dependence of the non-ergodicity factor of glassy sorbitol up to $T_{g}$. In any event, it is of some interest to add the $\alpha_{\chi}$ vs. $m$ data of sorbitol to the compilation reported in Ref. [5], as shown in Fig. 5. The point corresponding to sorbitol agrees with the general trend shown by Buchenau et al., suggesting that while a general correlation between $\alpha$ and $m$ seems to hold, the data of $\alpha_{\chi}$ flatten out to become basically fragility independent for systems with high fragility.

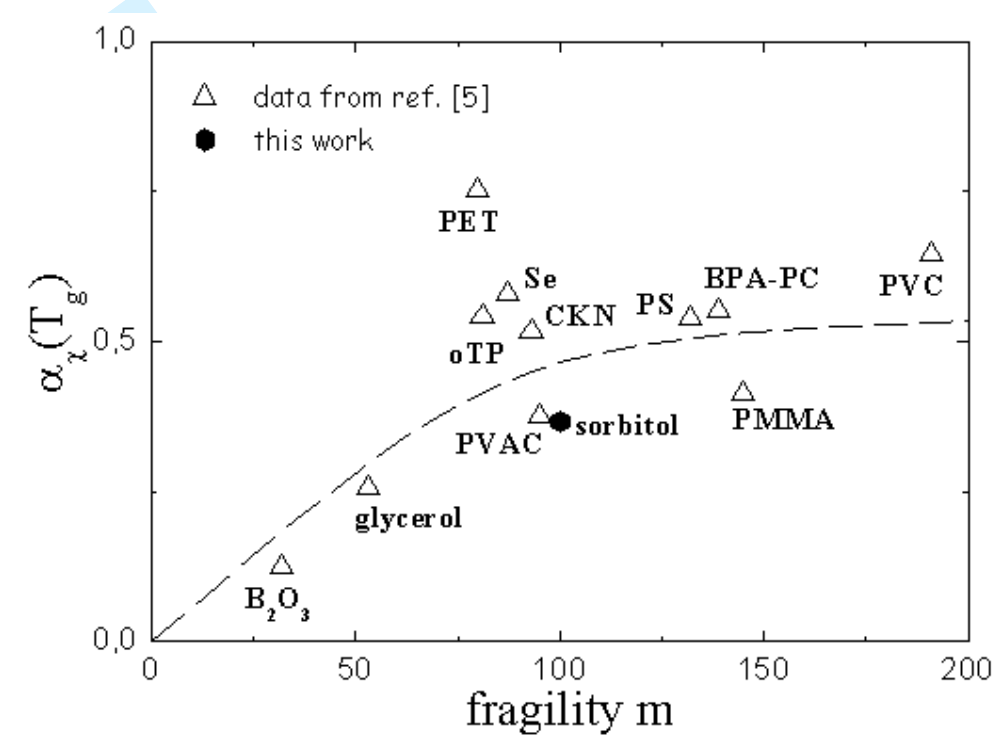

Figure 5. Correlation between the ratio $\alpha_{\chi}$ of vibrational to relaxational compressibility at $T_{g}$ and the fragility $m$. The empty symbols are taken from Ref. [5], while the full circle corresponds to sorbitol. The dashed line is a guide for the eye.

\section{Conclusions}

Brillouin light scattering measurements of the fragile glass-former sorbitol have been here presented. The data have been collected in the backscattering geometry over a wide temperature range in the glassy and supercooled liquid phase. The temperature dependence of the sound velocity and of the damping of the longitudinal acoustic excitations has been reported and discussed. The joint analysis of the present data and of PVT data available in the literature [9] has allowed us to derive the values of the two independent elastic moduli of the sorbitol glass. We show that the ratio of the bulk to shear modulus in sorbitol doesn't follow the linear dependence on the fragility found for several glasses by Novikov et al. [4]. In fact, for glassy sorbitol $B^{\prime} / G^{\prime}=1.73$, a value similar to that usually found in stronger systems such as $\mathrm{B}_{2} \mathrm{O}_{3}\left(B^{\prime} / G^{\prime}=1.95\right)$. Conversely, the ratio between vibrational and relaxational compressibility at the glass transition temperature is close to that of other fragile and very fragile systems. This compressibility ratio seems in fact to be correlated to the fragility though it becomes basically fragility independent for very fragile glasses. 
B.R. and G.M. acknowledge C. Alba-Simionesco, K. Niss and C. Dalle-Ferrier for suggesting the case of sorbitol.

\section{References}

[1] C.A. Angell, J. Non-Cryst. Solids (1985), 73, 1.

[2] T. Scopigno et al., Science (2003), 302, 849.

[3] V.N. Novikov, Y. Ding, A.P. Sokolov, Phys. Rev. E (2005), 71, 061501.

[4] V.N. Novikov, A.P. Sokolov, Nature (2004), 431, 961.

[5] U. Bucheanu, A. Wischnewski, Phys. Rev. B (2004), 70, 092201.

[6] L.-M. Wang, C.A. Angell, R. Richert, J. Phys. Chem. (2006), 125, 074505, and reference therein.

[7] R.A. Talja, H.H. Roos, Thermochim. Acta (2001), 380, 109.

[8] D. Fioretto, et al., Phys. Rev. B (1993), 22, 15286.

[9] M. Naoki, K. Ujita, S. Kashima, J. Phys. Chem. (1993), 97, 12356.

[10] T.H.K. Barron, J.G. Collins, G.K. White, Adv. Phys. (1980), 29, 609.

[11] E. Rat et al., Phys. Rev. B (2005), 72, 214204.

[12] J. Fabian, P.B. Allen, Phys. Rev. Lett. (1999), 82, 1478.

[13] S. Shamblin et al., J. Phys. Chem. B (1999), 103, 4113.

[14] A. Monaco, PhD Thesis (2006).

[15] K. Ito, C.T. Moynihan, C.A. Angell Nature (1999), 398, 492. 